\title{
Electromyographic Evaluation of Specific Elastic Band Exercises Targeting Neck and Shoulder Muscle Activation
}

\author{
Ying Gao ${ }^{1,2}{ }^{(0}$, Lars A. Kristensen ${ }^{3}$, Thomas S. Grøndberg ${ }^{3}$, Mike Murray ${ }^{3}$, Gisela Sjøgaard ${ }^{3}(0)$ \\ and Karen Søgaard $3,4, *$ (D) \\ 1 Department of Sports Science, College of Education, Zhejiang University, Hangzhou 310028, China; \\ yigao@zju.edu.cn \\ 2 Faculty of Sport and Health Sciences, University of Jyväskylä, 40700 Jyväskylä, Finland \\ 3 Department of Sports Science and Clinical Biomechanics, University of Southern Denmark, DK-5230 Odense, \\ Denmark; larsakristensen1@gmail.com (L.A.K.); thomas-stig@hotmail.com (T.S.G.); \\ mike_ster@hotmail.com (M.M.); gsjogaard@health.sdu.dk (G.S.) \\ 4 Department of Clinical Research, University of Southern Denmark, DK-5230 Odense, Denmark \\ * Correspondence: ksogaard@health.sdu.dk; Tel.: +45-6550-4409
}

Received: 12 December 2019; Accepted: 16 January 2020; Published: 21 January 2020

Featured Application: This electromyographic evaluation of elastic band exercises documents that the type and intensity of exercise played a role in the activity in three muscle groups of the neck and shoulder. However, importantly, shrugs and reverse flyes are simple to perform and also highly effective training exercises as they induced high muscle activity of both shoulder and neck muscles at 12RM. The practical implications of these findings are that these simple exercises can be preferred for training programs targeting neck and shoulder muscle either for increased general strength or as prevention for work related pain and disorders.

Abstract: Background: Specific strength training at a high intensity is effective in reducing work related neck/shoulder pain. However, it remains to be documented as to which exercises most specifically target neck and shoulder muscles at high activation level while using simple equipment as e.g., elastic bands. We hypothezised that selected exercises would specifically target the respective muscles, as follows: (1) shrugs and reverse flyes: the upper trapezius muscle, (2) cervical extension and lateral flexion: the upper neck extensor muscle, and (3) cervical flexion and rotation: the sternocleidomastoideus muscle. Methods: Eleven healthy males (25.9 \pm 1.4 years, BMI $24.3 \pm 1.4)$ with no neck/shoulder pain (VAS $=0$ ) performed the six exercises with elastic bands at 12RM (repetition maximum) and 20RM in a randomized order. Electromyography was bilaterally recorded from the three muscles and it was normalized to maximal voluntary activation (\%MVE). Exercises that evoke more than $60 \% \mathrm{MVE}$ were considered as high intensity activation. Results: High muscle activation level was attained during $12 \mathrm{RM}$ in the upper trapezius muscle during shrugs $(100.3 \pm 29.8 \% \mathrm{MVE})$ and reverse flyes $(91.6 \pm 32.8 \% \mathrm{MVE})$ and in the upper neck extensor muscle during cervical extension $(67.6 \pm 29.8 \% \mathrm{MVE})$ and shrugs $(61.9 \pm 16.8 \% \mathrm{MVE})$. In the sternocleidomastoideus muscle, the highest activity was recorded during cervical flexion $(51.7 \pm 16.4 \% \mathrm{MVE})$ but it did not exceed $60 \% \mathrm{MVE}$. The overall activity was $\sim 10 \%$ higher during $12 \mathrm{RM}$ when compared to 20RM. Conclusion: The simple exercises shrugs and reverse flyes resulted in high intensity activation of both the upper trapezius and neck extensors, while no exercises activated sternocleidomastoideus at high intensity.

Keywords: physical training intensity; therapeutic exercises; work related neck pain; musculoskeletal pain 


\section{Introduction}

Neck and shoulder pain are frequent in the general population and within several occupational groups that are characterized by monotonous work tasks that require the stability of the head and neck in constrained postures [1-6]. In often male dominated jobs, helmets add to the weight of the head increasing the demands on the neck muscles and a high prevalence of neck pain is reported among e.g., construction workers, helicopter, and fighter pilots [7-9]. Physical exercise-in particular specific high intensity strength training at the job-has been shown to effectively reduce musculoskeletal pain in the neck and shoulders [10-13].

In clinical practice, at work sites, and in home-based rehabilitation the use of conventional resistance training equipment, such as mascines or free weight, may not be a possibility, as it requires high financial cost and advanced facilities. Thus, it is important to identify more feasible alternatives that are easy to perform at the workplace and still effectively target muscles in the neck and shoulder regions. One alternative is the use of elastic resistance bands that has recently gained interest, because they are simple to handle, low in cost, and easy to transport. However, with elastic bands, it might specifically for the neck extensor muscles be difficult to add enough external load as resistance to achieve the requested high activity level. To attain this, a special headband has been invented for more specific, but also more complex, elastic band neck exercises. These complex exercises have been applied in training interventions of helicopter pilots [8]. However, the attendance rate was low, which was possibly due to the more complex head band exercises; and, in that study, it was not investigated whether the more complex exercises actually resulted in a higher activation for the specific muscles when compared to the more simple neck exercises, like shrugs and reverse flyes $[8,14]$. The aim of the present study was to assess whether simple exercises as compared to the more complex exercises may suffice for sufficient activation of the neck and shoulder muscles.

Regarding the level of sufficient exercise intensity in strength training programs, this is often defined as the percentage of maximal voluntary force exerted [15]. In practice, one repetition maximum (RM) weight is often used as reference for the maximal weight that can be lifted in an exercise for a given number of repetitions. Activation levels of $>60 \%$ of maximal voluntary contraction force (MVC) are generally recommended to attain a physiological effect of resistance training [15]. A higher force per contraction can be performed the lower the number of repetitions per set, e.g., at 1RM the performed force is close to $100 \%$ MVC, decreasing to around $60 \%$ MVC when performing $12 \mathrm{RM}$-depending highly on training status. In well trained persons $60 \%$ MVC might be repeated more times, e.g., corresponding to 20RM.

To access the activation level, electromyography (EMG) is commonly used to measure the activity of the specific muscles that are involved in an exercise [16,17]; the bilateral upper trapezius muscle (mUTR), upper neck extensor muscles (mUNE), and sternocleidomastoideus muscle (mSCM) were the muscle groups included here. In the prevention and treatment of neck and shoulder pain such EMG measurements can ensure that a given mode and intensity of training will activate the target muscles to the sufficient level [18]. In addition, EMG measures allow for exploratory analysis, such as the development of muscle fatigue during multiple repetitions that might be an indicator of the long-term effect on hypertrophy, as well as the reduced perception of pain. The myoelectrical manifestations used as an indication of muscle fatigue is an increase in the amplitude and a shift in the power spectrum towards lower frequencies [19-21]. Previous studies with advanced exercises have presented important knowledge for selecting the most adequate exercises to target specific health effects [16,17]. However, studies using simple exercises have similarly shown an effect on both pain relieve and increase in strength $[2,22]$. The present study will expand on this by using simple (shrugs, $\mathrm{SH}$, and reverse flyes, RF), as well as more advanced (cervical extension, CE and cervical flexion, CF), and quite difficult (cervical lateral flexion, $\mathrm{CL}$ and cervical rotation, $\mathrm{CR}$ ) neck/shoulder exercises and compare the activation in the muscles specifically targeted with each exercise at 20RM and 12RM.

The main hypothesis was that specific training exercises were requested to maximize the activation of the individual neck and shoulder muscles: with mUTR activation being the highest during $\mathrm{SH}$ and 
RF, mUNE during CE and CL, and mSCM during CF and CR. Further, it was hypothesized that the intensity of the exercises significantly affected muscle activity: 12RM, resulting in higher activity than 20RM during the specific training exercises. Finally, we hypothesized that the muscle activity in the concentric phase was higher than in the eccentric phase and fatigue development was present in each set of an exercise being shown as an increase in EMG amplitude and a decrease in EMG frequency in the targeted muscle groups.

\section{Materials and Methods}

\subsection{Study Design and Subjects}

This study was conducted over three separate days that were interspaced with at least three days with no exercises. On the first day participants were familiarized with the elastic band training exercises and practiced the proper technique under supervision. On the second day, individual loadings were determined for each training exercise during low intensity, high repetition sets (20RM), and during high intensity, moderate repetition sets (12RM). All of the exercises were performed while using concentric and eeccentric muscle contractions in a controlled manner, with slow to moderate lifting velocity corresponding to the execution in earlier intervention studies [16]. Subsequently, the actual duration of concentric and eccentric phases were measured and shown to have an equal duaration each of minimum three seconds. The length and color code of elastic bands were adjusted to reduce or increase the resistance to match 12RM and 20RM for each participant. The task was repeated until the participant was exhausted and/or not able to continue the exercise according to the basics of procedures in the literature to define the individual 12RM and 20RM loads for each exercise [15]. The participant was deemed not able to continue the exercise if making compensating adjustment in body posture or not obeying contraction time and range of movement. The exercise was repeated until the loading corresponding to 20RM and 12RM was determined. On the third day, the determined loadings for each participant were applied and all EMG measurements were performed, while the participant performed all exercises at the intensity of 20RM sets, followed by 12RM sets. The order of exercises was randomized and each exercise was separated by 3-minutes rest intervals to avoid a gradient of fatigue through the test protocol. At the end, isometric maximal voluntary contractions (MVC) were performed for the normalization of the EMG data. For each targeted muscle, three MVCs with a duration of $5 \mathrm{~s}$ and 1 minute rest between consecutive trials were performed to induce the maximal muscle activity in a seated position and according to standardized procedures described in details in a recent study [23]. In short, maximal activity in MUTR was obtained during resisted bilateral shoulder elevation, with resistance being placed above the acromions. Maximal activity for mUNE was obtained during a resisted cervical extension and for $\mathrm{mSCM}$ during a resisted cervical flexion with resistance being placed on the external occipital protuberance and above the eyebrows, respectively.

The subjects participating were a group of 11 healthy males (age $25.9 \pm 1.4$ years, height 183.6 $\pm 5.0 \mathrm{~cm}$, body mass $82.1 \pm 6.0 \mathrm{~kg}$, BMI $24.3 \pm 1.4$ ), with no pain in neck or shoulders (VAS $=0$ ). The inclusion of only males was justified by the aviation personell—who used these exercises in a previous RCT study-all being males [8]. All of the subjects were informed regarding the benefits and risks of the investigation prior to signing the institutionally approved informed consent document to participate in the study. The local Ethics Committee of Southern Denmark (S-20120121) approved the procedures in the study.

\subsection{Procedures}

\subsubsection{Electromyography (EMG)}

The activity of the neck and shoulder muscles was measured through a bipolar surface EMG configuration (Neuroline 720-01-K, Medicotest A/S, Ølstykke, Denmark). The electrodes (Ag/AgC1, Ambu Blue Sensor, N-00-S/25, Denmark) were positioned bilaterally, in accordance with the SENIAM 
recommendations and earlier studies [24-27]) with an inter-electrode distance of $20 \mathrm{~mm}$. The electrodes were placed (1) above the upper trapezius muscle (mUTR), $20 \%$ medially from the centerpoint between the lateral edge of the acromion and seventh cervical vertebra, (2) above the upper neck extensor (mUNE) muscle, on the most prominent part of the muscle at the level of the fourth cervical vertebra, and (3) above the sternocleidomastoideus muscle (mSCM), over the main muscle belly at the $1 / 3$ distance from the sternal notch to the mastoid process (Figure 1). Before affixing the electrodes, skin was shaved and cleaned with scrubbing gel (Acqua gel, Meditec, Parma, Italy) and 70\% alcohol, to effectively lower the impedance to less than $10 \mathrm{k} \Omega$.

\section{A}

B

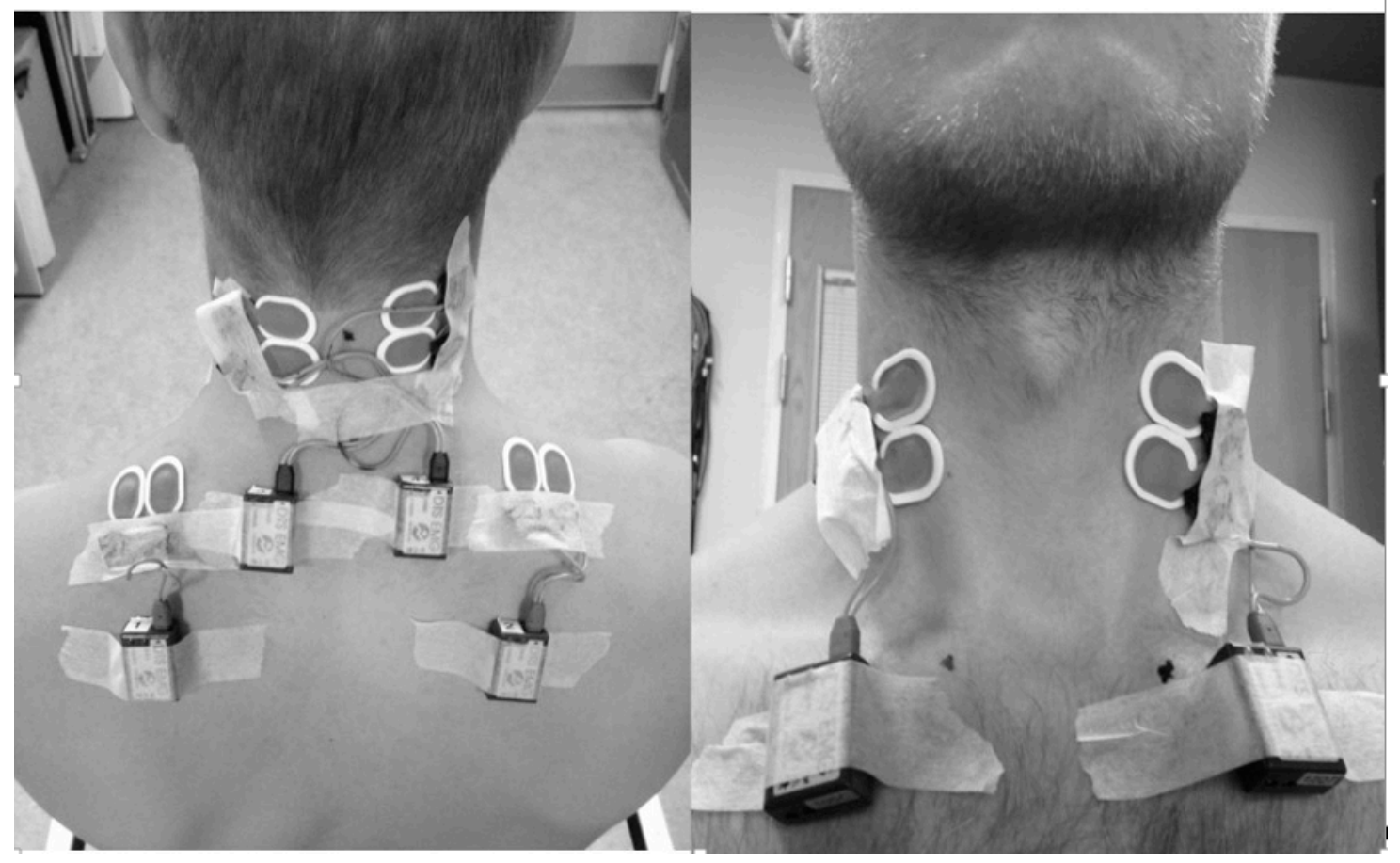

Figure 1. Bilateral electrode placement above the upper trapezius muscle (mUTR) and upper neck extensors muscles (mUNE) (A) and sternocleidomastoideus muscles (mSCM) (B).

The EMG signal was pre-amplified with a gain of 400 and then transferred through an EMG probe (Direct Transmission System (DTS), Noraxon Inc, Scottsdale, AZ, USA) to a belt-receiver (TELEMyo TM 2400T, DTS, Noraxon Inc, Scottsdale, AZ, USA) with a bandwidth of 10-500 Hz and a common mode rejection ratio better than $100 \mathrm{~dB}$. The signal was sampled at $1000 \mathrm{~Hz}$ while using a 16-bit A/D converter (DAQCard Al 16XE 50, National Instruments, Austin, TX, USA) and stored on a computer via the laboratory interface (CED 1401, Spike2 software, Cambridge Electronic Devices, Cambridge, UK).

\subsubsection{Elastic Band Training Exercises}

Six specifically tailored elastic band training exercises were performed targeting the neck and shoulder muscles. The choice of exercises was evidence-based and designed by an interdisciplinary team, including sports exercise training specialists, physical therapists, medical doctors, and chiropractors. The exercises were performed with a head harness (Neck Flex ${ }^{\circledR}$, Leesburg, VA, USA) while using different color-coded elastic resistance bands (Thera-Band ${ }^{\circledR}$, The Hygenic Corporation, Akron, OH, USA). The individual exercises are briefly presented below, as a detailed description can be found in an earlier publication [14] and video material is available online: Neck exercises.

In short the exercises are the following: 
(1) Shrugs (SH) were performed in standing position with the elastic band fixated under the feet and arms placed along the side of the body and shoulders relaxed. The head was held in an anatomically neutral position with eyes looking straight forward. During the exercise, shoulders were elevated as high as possible toward the ears against resistance and lowered again.

(2) Reverse Flyes (RF) were performed seated with the elastic band fixated under the feet and with a straight back. Participants were instructed to position their head in an anatomically neutral position, lean the trunk forward $\left(\sim 20-30^{\circ}\right)$, and place both arms pointing towards the floor. Elbows were kept in a static and slightly flexed position $\left(\sim 5^{\circ}\right)$. During the exercise, both of the arms were raised toward a horizontal level against resistance and lowered again.

(3) Cervical Extension (CE) was performed seated. The participants were instructed to keep a straight back, position their head in an anatomically neutral position, and lean the trunk forward $\left(\sim 20-30^{\circ}\right)$. Arms were held strait with the hands placed underneath the knees. An elastic band was stretched between the hands and front of the head harness. The exercise was performed with a low cervical spine flexion, followed by a low cervical spine extension (against resistance).

(4) Cervical Flexion (CF) was performed in the same way as during cervical extension, but the elastic band was stretched between a door anchor and the back of the head harness. During the exercise, the participants performed a low cervical spine flexion (against resistance), followed by a low cervical spine extension.

(5) Cervical Lateral flexion (CL) was performed standing erect with the head in an anatomically neutral position. One hand was placed horizontally against a wall and an elastic band was stretched between the hand and side of the head harness. The exercise was performed with a low lateral spine flexion against resistance, followed by a low lateral spine extension. The exercise was performed for the right and left side, respectively.

(6) Cervical rotation (CR) was performed seated with a straight back and trunk leaned forward $\left(\sim 20^{\circ}\right)$. The head was held in an anatomically neutral position and rotated approximately $45^{\circ}$ to either the right or left side. An elastic band was stretched between the head harness and a door anchor. Keeping a static upper body, the hips were flexed and the body flexed (against resistance), followed by an extension. The exercise was performed to the right and left side.

\subsection{Outcomes}

Analyses of the EMG signals were the outcome data. All data analysis was performed while using a custom-made script in Matlab (MathWorks Inc. USA) and Hedera 2.0 (University of Southern Denmark, Denmark). The peak EMG amplitude was calculated by root mean square (RMS) in a $500 \mathrm{~ms}$ moving window. For each repetition during exercises, the peak RMS was normalized to the peak RMS that was obtained during the MVC of each muscle as percentage of maximal voluntary EMG signal (\%MVE) in standardized postures [23]. The 3rd, 4th, and 5th repetition of each exercise were averaged and used as the muscle activation for the exercises. The EMG activity in the concentric and eccentric phases of the muscle contraction was analyzed for each muscle during the exercise, resulting in the highest activation. The concentric and eccentric phases of each exercise were marked as an event in a separate channel in the Spike2 software, during each recording, As each repetition was performed with a $3 \mathrm{~s}$ concentric and $3 \mathrm{~s}$ eccentric phase, the midpoint was used to divide these phases. Additionally, the mean power frequency (MPF) was calculated in the last $1000 \mathrm{~ms}$ of the concentric phase of each contraction for the muscle groups during the exercise that represents the highest activation during the contraction.

\subsection{Statistical Analysis}

All of the data are presented as mean \pm SD. The Q-Q plot and the Shapiro-Wilk test with skewness were used to test for normal distribution of data. Differences between left and right muscles during the symmetrical exercises $\mathrm{SH}, \mathrm{RF}, \mathrm{CE}$, and $\mathrm{CF}$, or between ipsilateral and contralateral muscles during $\mathrm{CL}$ and $\mathrm{CR}$ performed towards left and right side were examined while using paired-t test. In the case of no difference, the mean value of the two sides was used. If significant differences were found, 
the side specific values are reported and the highest used to represent the muscle activation level for that exercise. Regarding the main hypotheses of the muscle activity (\%MVE) for each muscle group, one way repeated measures ANOVA was used to compare the different exercises (SH, RF, CE, $\mathrm{CF}, \mathrm{CL}$, and CR). If the assumption of Sphericity was violated, the Greenhouse-Geisser correction was used. Planned contrasts were used to localize the differences between the exercise, resulting in the highest activation of specific muscle group and the other exercises. Paired t-test was used for testing possible differences between the two exercise intensities. Furthermore, paired t-test was used to compare muscle activity in the concentric phase and eccentric phase of each muscle group. The fatigue development was tested by paired t-test to compare the third repetition and the last one repetition in normalized EMG amplitude (\%MVE) and EMG frequency (MPF). A substantial addition in muscle activation level of at least $20 \%$ was requested in order to recommend an advanced, instead of a simple, exercise. This level is well above the SD of approx. $10 \%$ in mean level of trapezius activation both found within groups and variability of repeated EMG recordings during high intensity contractions, see, for instance, mean activation levels for trapezius in [17]. A power analysis showed that eight subjects would be sufficient to reveal a difference in muscle activation level of $20 \%$. Within the recruiting period, we managed to recruit 11, who all conducted the full protocol. All of the results were analyzed using SPSS 16.0 statistical software (SPSS Inc, Chicago, IL, USA). A probability level of $p<0.05$ (two-tailed) was considered to be statistically significant.

\section{Results}

Table 1 presents the muscle activation levels for the six exercises at two intensities. There were no differences between left and right side of muscles during the symmetrical exercises and no difference between ipsilateral and contralateral when the exercises CL and CR were performed to right versus left side. Therefore, the data were averaged over left and right muscles or ipsilateral and contralateral muscles, except during CL for mUNE and $\mathrm{mSCM}$. This is due to the higher muscle activity during CL in ipsilateral than contralateral for mUNE $(30.2 \% \pm 13.6 \%$ vs. $12.8 \% \pm 6.8 \%, p<0.001$, for $20 \mathrm{RM}$, and $45.5 \% \pm 14.6 \%$ vs. $17.2 \% \pm 7.1 \%, p<0.001$, for $12 \mathrm{RM})$ and for $\operatorname{mSCM}(25.6 \% \pm 7.7 \%$ vs. $11.6 \% \pm 6.3 \%$, $p=0.001$, for $20 \mathrm{RM}$, and $41.5 \% \pm 14.6 \%$ vs. $15.3 \% \pm 8.4 \%, p=0.001$, for $12 \mathrm{RM})$. Therefore, for CL the higher value of \%MVE in ipsilateral of mUNE and $\mathrm{mSCM}$ were used as the results (Table 1).

Table 1. Mean muscle activity (\%MVE) for representative muscle groups shown for each exercise at $12 \mathrm{RM}$ and 20RM intensities. Values are presented as mean $\pm \mathrm{SD}$. Values $>60 \%$ MVE are marked in bold. Italic values are unilateral data, see text.

\begin{tabular}{lccccccc}
\hline \multirow{2}{*}{ Muscle } & \multirow{2}{*}{ Intensity } & \multicolumn{7}{c}{ Exercise } \\
\cline { 3 - 7 } & & SH & RF & CE & CF & CL & CR \\
\hline \multirow{2}{*}{ mUTR } & $20 R M$ & $\mathbf{7 8 . 8} \pm \mathbf{2 4 . 0}$ & $\mathbf{7 5 . 9} \pm \mathbf{3 0 . 7}$ & $3.4 \pm 1.8$ & $1.9 \pm 1.3$ & $8.1 \pm 4.6$ & $3.7 \pm 2.3$ \\
& $12 R M$ & $\mathbf{1 0 0 . 3} \pm \mathbf{2 9 . 8}$ & $\mathbf{9 1 . 6} \pm \mathbf{3 2 . 8}$ & $4.8 \pm 2.5$ & $2.4 \pm 2.0$ & $9.1 \pm 4.1$ & $4.3 \pm 2.8$ \\
\hline \multirow{2}{*}{ mUNE } & $20 \mathrm{RM}$ & $45.7 \pm 16.7$ & $39.1 \pm 22.3$ & $50.9 \pm 25.8$ & $7.6 \pm 3.2$ & $30.2 \pm 13.6$ & $10.4 \pm 4.6$ \\
& $12 \mathrm{RM}$ & $\mathbf{6 1 . 9} \pm \mathbf{1 6 . 8}$ & $55.4 \pm 16.3$ & $\mathbf{6 7 . 6} \pm \mathbf{2 9 . 8}$ & $8.5 \pm 3.8$ & $45.5 \pm 14.6$ & $12.0 \pm 4.7$ \\
\hline \multirow{2}{*}{ mSCM } & $20 \mathrm{RM}$ & $16.7 \pm 13.5$ & $14.2 \pm 8.9$ & $15.1 \pm 11.3$ & $44.8 \pm 16.6$ & $25.6 \pm 7.7$ & $28.8 \pm 9.9$ \\
& $12 \mathrm{RM}$ & $26.2 \pm 16.5$ & $22.9 \pm 14.8$ & $10.1 \pm 6.3$ & $51.7 \pm 16.4$ & $41.5 \pm 14.6$ & $42.5 \pm 11.6$ \\
\hline
\end{tabular}

The repeated measures ANOVAs revealed significantly different muscle activation between the types of exercise for: $\operatorname{mUTR}(\mathrm{F}(1.099,10.987)=97.622, p<0.001), \operatorname{mUNE}(\mathrm{F}(2.359,23.59)=29.038$, $p<0.001)$, and $\operatorname{mSCM}(F(5,50)=18.045, p<0.001)$, respectively. Figure 2 presents the contrast of muscle activity during the exercise, which induced the highest activation with other exercises. For mUTR, SH induced highest activity, and this was significantly higher than all of the remaining exercises, except RF. For mUNE, CE induced the highest activity and this was higher than during CF, CL and CR, but not significantly different from SH, and RF. For mSCM, CF induced the highest activity and this was higher than in all the remaining exercises. Furthermore, when compared with 20RM, the mean muscle activity of all exercises was significantly higher (relative increase $34 \%$ ) during 12RM. For the 
specific muscle groups the relative increase were $26 \%$ in mUTR $(p<0.001), 41 \%$ in mUNE $(p<0.001)$, and $35 \%$ in $\operatorname{mSCM}(p<0.001)$. (Figure 3$)$.

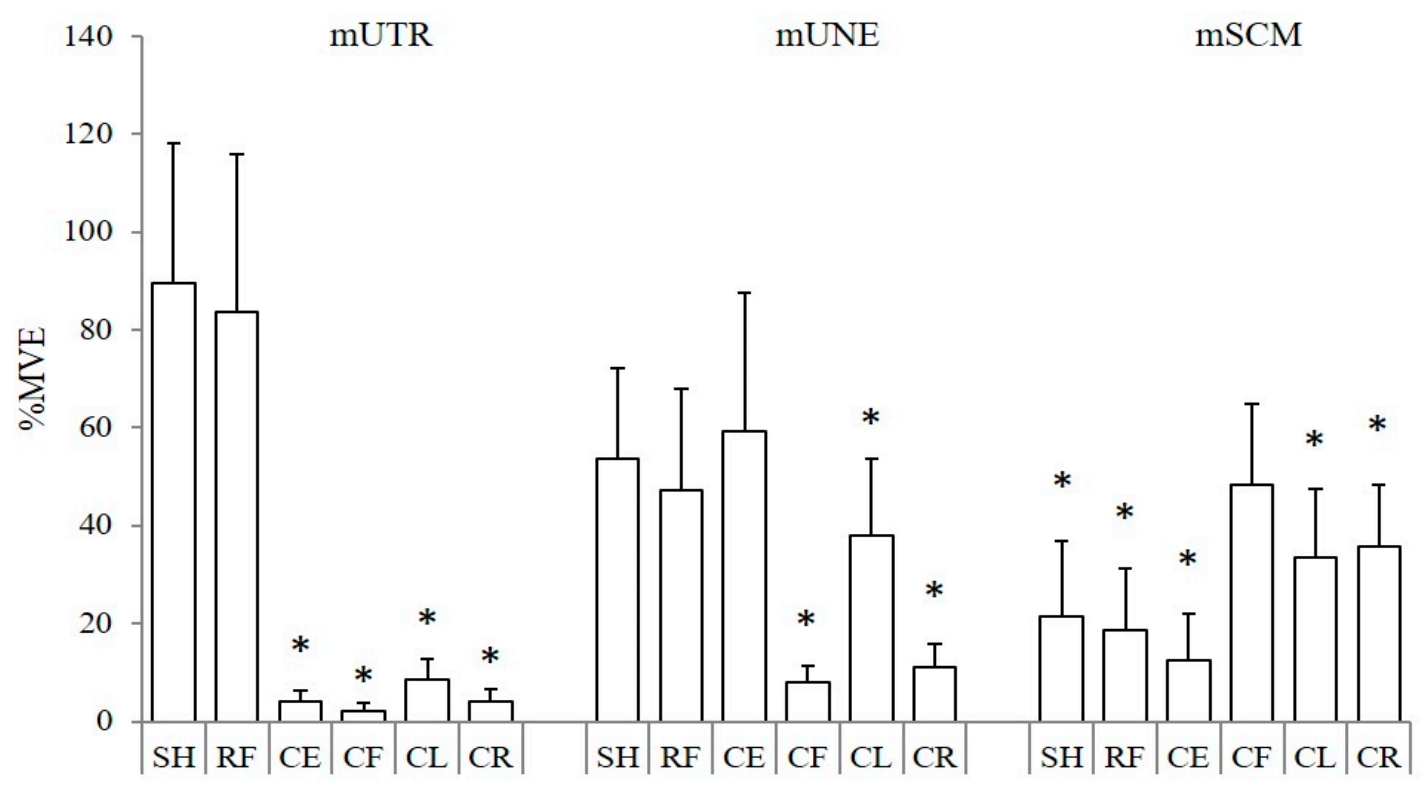

Figure 2. The muscle activity of the upper trapezius muscle (mUTR), upper neck extensors muscles (mUNE) and sternocleidomastoideus muscles (mSCM) were compared in six different types of exercises. The highest muscle activation (\%MVE) was found during SH for mUTR, during CE for mUNE and during CF for mSCM. * marks exercises with significantly lower activation than the exercise inducing the highest muscle activation.

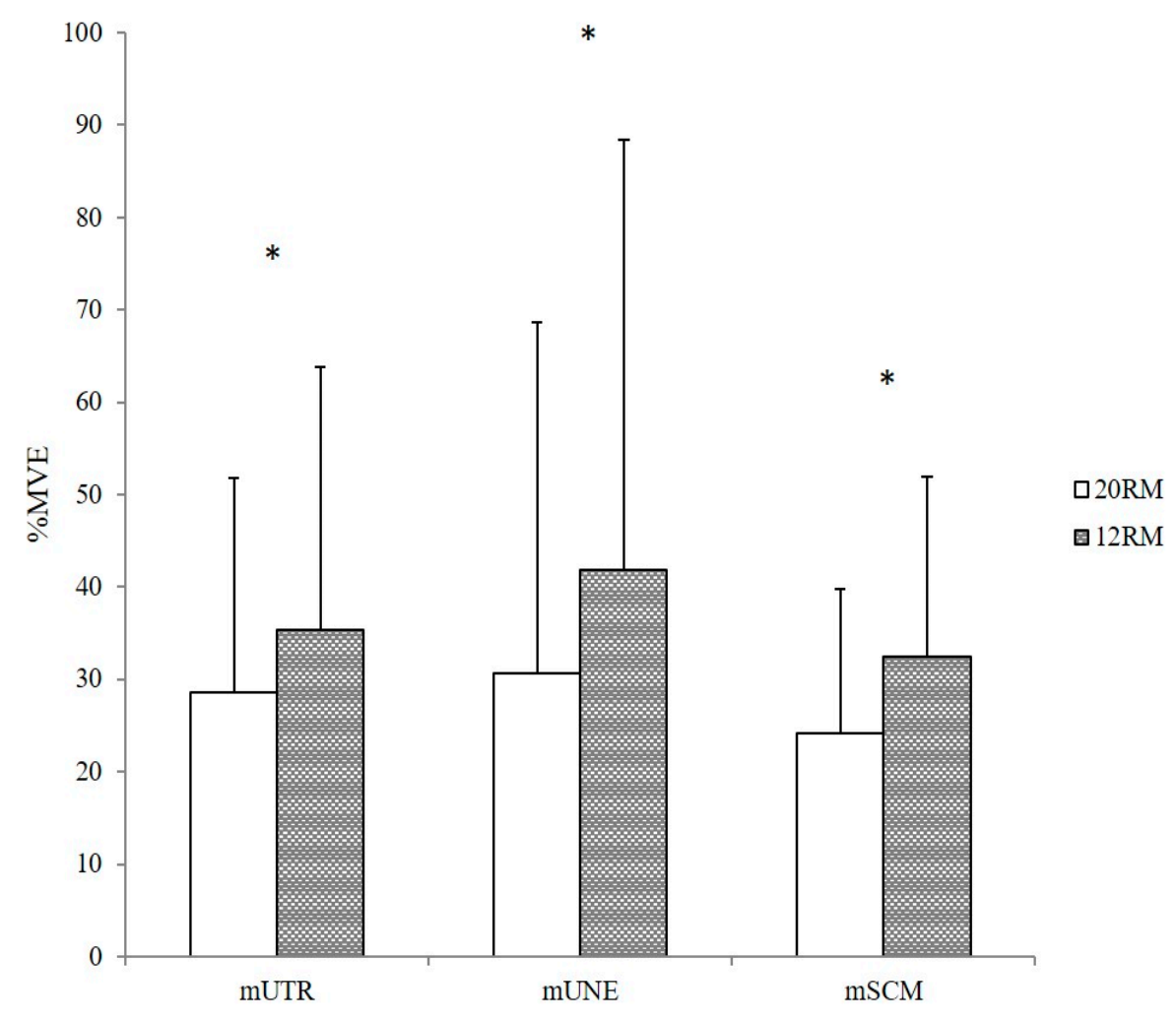

Figure 3. Mean muscle activity (\%MVE) for all exercises in the upper trapezius muscle (mUTR), upper neck extensors muscles (mUNE), and sternocleidomastoideus muscles mSCM during intensities of 12RM and 20RM. * indicates significant difference between training intensities. 
Analysis while considering activity separately in the concentric and eccentric contraction modes showed that the muscle activity of mUTR, mUNE, and $\mathrm{mSCM}$ in the concentric phase when compared to the eccentric phase was significantly increased by relative $75 \%$ for $\mathrm{SH}(p<0.001), 32 \%$ for $\mathrm{CE}$ $(p=0.009)$, and $22 \%$ for CF $(p=0.002)$ (Figure 4$)$.

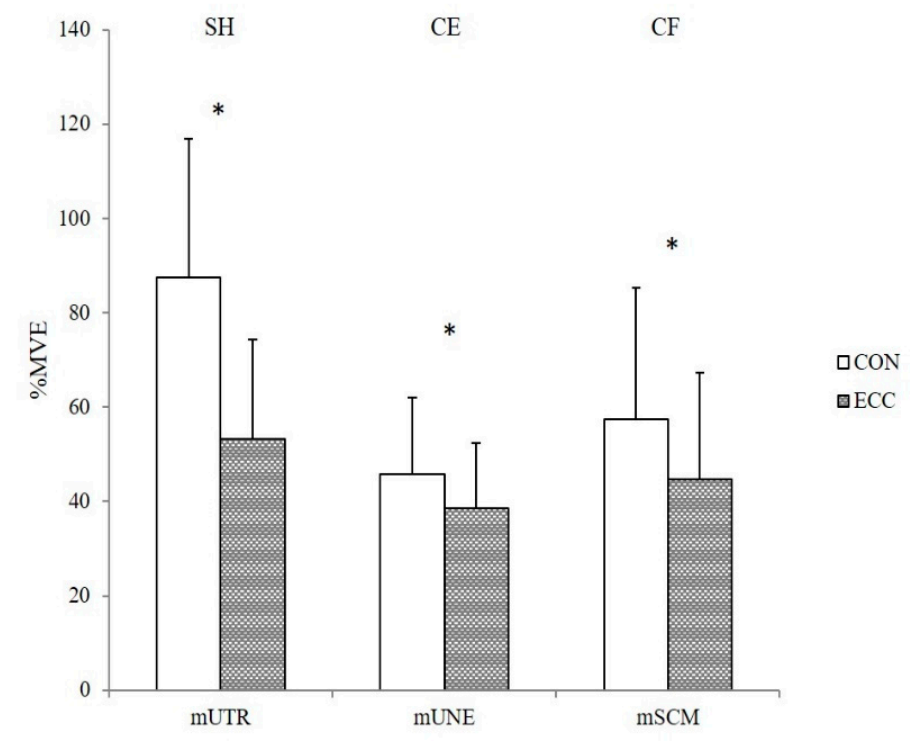

Figure 4. The muscle activity (\%MVE) of the upper trapezius muscle (mUTR), upper neck extensors muscles (mUNE), and sternocleidomastoideus muscles (mSCM) in the concentric phase (CON) and eccentric phase (ECC) during shugs (SH), cervical extension (CE), and cervical flexion (CF), respectively. * Indicates significant difference between CON and ECC within exercise.

Analysis of fatigue development showed that, in each set of an exercise, a significant increase occurred in \%MVE and a significant decrease occurred in EMG frequency (MPF) during SH, CE, and $\mathrm{CF}$ for each muscle groups of mUTR, mUNE, and mSCM, respectively (Table 2).

Table 2. The fatigue development of mean muscle activity (\%MVE) and mean power frequency (MPF) for the upper trapezius muscle (mUTR), upper neck extensors muscles (mUNE), and sternocleidomastoideus muscles mSCM at intensities of 12 and 20RM. \#Values are presented as mean \pm SD for the exercises shrugs (SH), cervical extension (CE), and cervical extension (CF) that resulted in the highest activation of each muscle.

\begin{tabular}{lcccccccc}
\hline \multirow{2}{*}{ Muscle } & \multirow{2}{*}{ Exercise } & \multirow{2}{*}{$\begin{array}{c}\text { Fatigue } \\
\text { Development }\end{array}$} & \multicolumn{3}{c}{ 20RM } & & \multicolumn{2}{c}{ 12RM } \\
\cline { 4 - 8 } & & & Rep 3. & Rep 19. & $p$-Values & Rep 3. & Rep 11. & $p$-Values \\
\hline \multirow{2}{*}{ mUTR } & \multirow{2}{*}{ SH } & \%MVC & $81.2 \pm 27.1$ & $95.6 \pm 25.6$ & 0.001 & $94.3 \pm 31.4$ & $114.1 \pm 30.2$ & $<0.001$ \\
& & MPF & $78.5 \pm 5.9$ & $69.8 \pm 8.2$ & $<0.001$ & $80.3 \pm 12.0$ & $71.6 \pm 10.2$ & $<0.001$ \\
\hline \multirow{2}{*}{ mUNE } & \multirow{2}{*}{ CE } & \%MVC & $50.7 \pm 26.2$ & $67.5 \pm 33.7$ & 0.006 & $64.5 \pm 28.7$ & $82.4 \pm 33.7$ & 0.010 \\
& & MPF & $66.0 \pm 7.6$ & $57.2 \pm 8.9$ & 0.004 & $63.6 \pm 9.4$ & $57.6 \pm 10.6$ & 0.005 \\
\hline \multirow{2}{*}{ mSCM } & \multirow{2}{*}{$\mathrm{CF}$} & \%MVC & $43.9 \pm 17.4$ & $53.5 \pm 20.7$ & 0.016 & $51.4 \pm 17.1$ & $65.0 \pm 21.5$ & 0.027 \\
& & MPF & $104 \pm 14.4$ & $95.2 \pm 14.4$ & 0.005 & $104.0 \pm$ & $99.1 \pm 12.7$ & 0.012 \\
\hline
\end{tabular}

\section{Discussion}

\subsection{Main Finding}

Specific activation patterns were identified in the three neck/shoulder muscles that were studied, in accordance with the hypothesis exercise type. Interestingly, the simple exercises, $\mathrm{SH}$ and RF, elicited muscle activities of $>60 \%$ MVE in mUNE as well as in mUTR. The more advanced exercise, CE, showed 
numerically slightly higher-but not significantly—activation of mUNE, and CF produced the highest activity in $\mathrm{mSCM}$, but this did not attain $>60 \%$ MVE neither during 20RM nor 12RM and can therefore, not be recommend in case of $\mathrm{mSCM}$ insufficiencies. The two muscle groups most often attracting work related pain are mUTR and mUNE [1,3-5]. It is therefore interesting that both muscles can be intensively activated by two simple exercises (SH and RF), as this might potentially counteract the work related pain. The CL and CR, which are the most difficult exercises to perform correctly, did not elicit significantly higher percentages of muscle activation than any of the other exercises and can therefore be disregarded, unless for extra training variation. Thereby, our main hypothesis was rejected.

Earlier studies have reported significantly higher activation levels of mUTR during SH than RF $[17,28]$, but this was not the case in the present study. The similar activation levels of mUTR during $\mathrm{SH}$ and RF may have great clinical importance due to the difference in force resistance that is required for performing the exercises. When performing the $\mathrm{SH}$ exercise for all subjects, a combination of three high resistance elastic bands were needed, whereas only one low resistance elastic band was used during RF. Thus, the SH exercise requires significant grip strength and core stability, which might be challenging for some populations. The mUTRs role as a scapula up- and outward rotator in combination with the significant external moment arm during the RF exercise may explain the high muscle activation in spite of low external resistance. Therefore, the RF exercise might, for some populations, be a more appropriate choice of exercise as compared to the $\mathrm{SH}$.

The activity of mUNE was not significantly higher in CE compared to SH and RF. To our knowledge this is the first study to demonstrate high activity of neck muscles during SH and RF. This observation implies that the use of sophisticated exercises might not be needed as simple exercises effectively can activate both neck and shoulder muscles. This finding is supported by an earlier finding on high $(>60 \%$ MVE) muscle activity of $\mathrm{m}$. splenius capitis during lateral raise, which is similar to the RF that was used in the present study [29]. As splenius capitis is a part of the upper neck extensor muscle group, it will contribute to the gross activity measured as mUNE in the present study. Based on functional anatomy, the cervical spine will be forced in a protracted position during $\mathrm{SH}$ and RF, when performed with an external loading [30-32]. The neck muscles must be activated in order to stabilize and keep a neutral positio. This essential role of neck stability during $\mathrm{SH}, \mathrm{RF}$, and lateral raise may thus be a possible explanation for the high muscle activity in neck extensor muscle group. In this regard, it must be noted that the stabilizing effect arrives from static muscle contractions and the subsequent physiological effect might not be the same as from dynamic muscle contractions during the CE exercise [33-35]. Studies using wire electrodes to separate activity in the deep and superficial neck extensor muscles have pointed out the importance of activity specifically in the deep extensor muscles for patients with chronic neck pain [36]. Since surface EMG records from all of the underlying muscles, we cannot rule out that the same activity in $\mathrm{SH}$ and $\mathrm{CE}$ represent different contribution from the neck extensor muscles that may be of importance for specific patient groups. However, this distinction might not be equally important for the purpose of general strengthening of the neck extensors to relieve work related pain.

\subsection{Contraction Intensities}

In general, it is assumed that muscle activation increases with increased external load. Our hypothesis regarding a higher intensity during 12RM as compared 20RM was confirmed. We found that the EMG activity increased by $9 \%$ MVE from an overall mean of $28 \%$ MVE at 20RM to 37\% MVE at 12RM. This corresponds to the estimated increase from $60 \%$ of $1 \mathrm{RM}$ at $20 \mathrm{RM}$ to the $70 \%$ of $1 \mathrm{RM}$ at 12RM. However, this general increase consisted of largely different increases for each of the targeted muscles. The muscles have a rather different function in each exercise and a high degree of co-contraction occurring in the neck region. Only for the MUTR would the 20RM evoke intensities $>60 \%$ MVE that is normally considered to be most efficient for a training effect. From a pain reduction perspective, the high intensive exercises have been shown to be most effective and, therefore, the 12RM might be the best gain for the work time invested into exercises at the workplace $[11,18]$. 


\subsection{Muscle Activation Patterns}

The exercises included a concentric and an eccentric phase and EMG recordings clearly demonstrated that the highest activation occurred in the concentric phase, which is in concert with general physiological findings of the recruitment pattern explained by the force-velocity relationship $[37,38]$. Elastic band training exercises has been shown to compare well to activation patterns seen during traditional dumbbell exercises [29] and this underpins that elastic band exercise training might well substitute training with more advanced equipment. EMG recording also demonstrated that, during each set of contractions, significant muscle fatigue developed in all recorded muscle groups. In spite of the difference in activation level between the 12RM and 20RM in the first contraction of the sets then at the last contraction of the 12RM and 20RM both attained levels $>60 \% \mathrm{MVE}$, which may potentially evoke an increase in muscle capacity [15]. Hereby, the present hypotheses regarding the contraction mode and fatigue EMG patterns were confirmed.

\subsection{Study Strenghts and Limitations}

We consider this study as a first step in the investigation of recommendable training and rehabilitation exercises for the neck/shoulder muscle based on elastic bands. The strengths of the study is the direct measures using EMG to assess the activation of representative neck muscle groups and instead of subjective assessment. Additionally, the design of the study using randomization of the exercises is a strength, since this ensures that the fatigue development within each type of exercise is not biased by its timewise performance.

The limitations of present study relate to the population only including a small group of males similar to majority of employees in the job groups specifically challenged with high exposures of the neck muscles due to helmet wearing or high accelerations (fighter pilots, helicopter pilots, construction workers, etc.). Additionally, the population consist of healthy subjects without serious neck/shoulder disorders. Therefore, extrapolation of our results to patients with neck/shoulder injury should be undertaken with caution. We cannot conclude that patients suffering from neck/shoulder pain will show similar muscle activation patterns when performing the exercises that are studied here.

Furthermore, EMG activity during the dynamic exercises was normalized to measurements of EMG activity during static MVCs to quantify the level of muscle activation. Given the inherent methodological limitations that are associated with surface EMG, only a rough estimate of the absolute level of muscle activation can be inferred while using this method. Fine wire electrodes for some of the deeper layers of neck extensor muscles could have further detailed the contribution of activity from each of the neck extensors. Finally, more muscles and contraction intensities could be studied to give an even more complete activation pattern for optimal recommendations of training exercises.

\subsection{Future Perspectives}

While considering the limitations above, future studies should include participants with work-related neck pain in a randomized controlled trial preferably designed with three groups: (A) training only using the simple exercises, (B) training with a variety of exercises, including the advanced and difficult exercises, and (C) a control group. Importantly the training volume, progression, undulation, and duration should be similar for groups $\mathrm{A}$ and $\mathrm{B}$, as we have exemplified in previous studies $[9-11,13]$. The primary outcome should be neck pain testing the hypothesis that groups A and B would have equally positive effects. Additionally, secondary outcomes should be included, like muscle strength and adherence to the exercises; the latter in particular to test the hypothesis implied in the present study that adherence for group A would be larger that for group B.

\section{Conclusions}

The simple exercises (SH and RF) were highly effective, as they induced high muscle activation level of both shoulder and neck muscles and induced fatigue within the set of exercises. The more 
advanced neck exercises (CE and CF) and quite difficult exercises (CL and CR) did not, in general, add significantly higher muscle activation levels. The practical implications of these findings are that these simple exercises can be preferred for self-administered training programs-e.g., at the worksite- targeting neck and shoulder muscle either for increased general strength or as prevention for work related pain and disorders.

Author Contributions: Responsible for the conceptualization of the study were, M.M., G.S. and K.S.; the methodology, software, and validation was taken care of by M.M., L.A.K. and T.S.G.; formal analysis, M.M., L.A.K. and T.S.G.; investigation and experimental work, M.M., L.A.K. and T.S.G.; resources, G.S., K.S. and Y.G.; data curation and statistics, Y.G.; writing—original draft preparation, Y.G.; writing-review and editing, all authors.; visualization, Y.G., L.A.K. and T.S.G.; supervision, G.S. and K.S.; project administration, K.S. All authors have read and agreed to the published version of the manuscript.

Funding: This research received no external funding.

Conflicts of Interest: The authors declare no conflict of interest.

\section{References}

1. Sjøgaard, G.; Lundberg, U.; Kadefors, R. The role of muscle activity and mental load in the development of pain and degenerative processes at the muscle cell level during computer work. Eur. J. Appl. Physiol. 2000, 83, 99-105. [CrossRef]

2. Hagberg, M.; Harms-Ringdahl, K.; Nisell, R.; Hjelm, E.W. Rehabilitation of neck-shoulder pain in women industrial workers: A randomized trial comparing isometric shoulder endurance training with isometric shoulder strength training. Arch. Phys. Med. Rehabil. 2000, 81, 1051-1058. [CrossRef]

3. Unge, J.; Ohlsson, K.; Nordander, C.; Hansson, G.A.; Skerfving, S.; Balogh, I. Differences in physical workload, psychosocial factors and musculoskeletal disorders between two groups of female hospital cleaners with two diverse organizational models. Int. Arch. Occup. Environ. Health 2007, 81, 209-220. [CrossRef]

4. Van den Heuvel, S.G.; van der Beek, A.J.; Blatter, B.M.; Bongers, P.M. Do work-related physical factors predict neck and upper limb symptoms in office workers? Int. Arch. Occup. Environ. Health 2006, 79, 585-592. [CrossRef]

5. Van den Oord, M.H.; De Loose, V.; Meeuwsen, T.; Sluiter, J.K.; Frings-Dresen, M.H. Neck pain in military helicopter pilots: Prevalence and associated factors. Mil. Med. 2010, 175, 55-60. [CrossRef]

6. Adam, J. Results of NVG-Induced Neck Strain Questionnaire Study in CH-146 Griffon Aircrew; DRDC Toronto, Defence R\&D Canada: Toronto, ON, Canada, 2004.

7. Lange, B.; Toft, P.; Myburgh, C.; Sjøgaard, G. Effect of targeted strength, endurance, and coordination exercise on neck and shoulder pain among fighter pilots: A randomized-controlled trial. Clin. J. Pain 2013, 29, 50-59. [CrossRef]

8. Murray, M.; Lange, B.; Nornberg, B.R.; Sogaard, K.; Sjogaard, G. Self-administered physical exercise training as treatment of neck and shoulder pain among military helicopter pilots and crew: A randomized controlled trial. BMC Musculoskelet. Disord. 2017, 18, 147. [CrossRef]

9. Gram, B.; Andersen, C.; Zebis, M.K.; Bredahl, T.; Pedersen, M.T.; Mortensen, O.S.; Jensen, R.H.; Andersen, L.L.; Sjøgaard, G. Effect of training supervision on effectiveness of strength training for reducing neck/shoulder pain and headache in office workers: Cluster randomized controlled trial. BioMed Res. Int. 2014, 2014, 693013. [CrossRef]

10. Blangsted, A.K.; Sogaard, K.; Hansen, E.A.; Hannerz, H.; Sjogaard, G. One-year randomized controlled trial with different physical-activity programs to reduce musculoskeletal symptoms in the neck and shoulders among office workers. Scand. J. Work. Environ. Health 2008, 34, 55-65. [CrossRef]

11. Andersen, L.L.; Kjaer, M.; Sogaard, K.; Hansen, L.; Kryger, A.I.; Sjogaard, G. Effect of two contrasting types of physical exercise on chronic neck muscle pain. Arthritis Rheum. 2008, 59, 84-91. [CrossRef]

12. Zebis, M.K.; Andersen, L.L.; Pedersen, M.T.; Mortensen, P.; Andersen, C.H.; Pedersen, M.M.; Boysen, M.; Roessler, K.K.; Hannerz, H.; Mortensen, O.S.; et al. Implementation of neck/shoulder exercises for pain relief among industrial workers: A randomized controlled trial. BMC Musculoskelet. Disord. 2011, 12, 205. [CrossRef] 
13. Dalager, T.; Bredahl, T.G.; Pedersen, M.T.; Boyle, E.; Andersen, L.L.; Sjøgaard, G. Does training frequency and supervision affect compliance, performance and muscular health? A cluster randomized controlled trial. Man. Ther. 2015, 20, 657-665. [CrossRef] [PubMed]

14. Murray, M.; Lange, B.; Nornberg, B.R.; Sogaard, K.; Sjogaard, G. Specific exercise training for reducing neck and shoulder pain among military helicopter pilots and crew members: A randomized controlled trial protocol. BMC Musculoskelet. Disord. 2015, 16, 198. [CrossRef] [PubMed]

15. Ratamess, N.A.; Alvar, B.A.; Evetoch, T.K.; Housh, T.J.; Kibler, W.B.; Kraemer, W.J.; Triplett, N.T. American College of Sports Medicine position stand. Progression models in resistance training for healthy adults. Med. Sci. Sports Exerc. 2009, 41, 687-708. [CrossRef]

16. Andersen, C.H.; Zebis, M.K.; Saervoll, C.; Sundstrup, E.; Jakobsen, M.D.; Sjogaard, G.; Andersen, L.L. Scapular muscle activity from selected strengthening exercises performed at low and high intensities. J. Strength Cond. Res. 2012, 26, 2408-2416. [CrossRef]

17. Andersen, L.L.; Kjaer, M.; Andersen, C.H.; Hansen, P.B.; Zebis, M.K.; Hansen, K.; Sjogaard, G. Muscle activation during selected strength exercises in women with chronic neck muscle pain. Phys. Ther. 2008, 88, 703-711. [CrossRef]

18. Chen, X.; Coombes, B.K.; Sjogaard, G.; Jun, D.; O'Leary, S.; Johnston, V. Workplace-Based Interventions for Neck Pain in Office Workers: Systematic Review and Meta-Analysis. Phys. Ther. 2018, 98, 40-62. [CrossRef]

19. Beck, T.W.; Stock, M.S.; Defreitas, J.M. Shifts in EMG spectral power during fatiguing dynamic contractions. Muscle Nerve 2014, 50, 95-102. [CrossRef]

20. Farina, D.; Merletti, R.; Enoka, R.M. The extraction of neural strategies from the surface EMG: An update. J. Appl. Physiol. 2014, 117, 1215-1230. [CrossRef]

21. Enoka, R.M.; Duchateau, J. Muscle fatigue: What, why and how it influences muscle function. J. Physiol. 2008, 586, 11-23. [CrossRef]

22. Ris, I.; Sogaard, K.; Gram, B.; Agerbo, K.; Boyle, E.; Juul-Kristensen, B. Does a combination of physical training, specific exercises and pain education improve health-related quality of life in patients with chronic neck pain? A randomised control trial with a 4-month follow up. Man. Ther. 2016, 26, 132-140. [CrossRef] [PubMed]

23. Murray, M.; Lange, B.; Chreiteh, S.S.; Olsen, H.B.; Nornberg, B.R.; Boyle, E.; Sogaard, K.; Sjogaard, G. Neck and shoulder muscle activity and posture among helicopter pilots and crew-members during military helicopter flight. J. Electromyogr. Kinesiol. 2016, 27, 10-17. [CrossRef] [PubMed]

24. Holtermann, A.; Roeleveld, K.; Mork, P.J.; Gronlund, C.; Karlsson, J.S.; Andersen, L.L.; Olsen, H.B.; Zebis, M.K.; Sjogaard, G.; Sogaard, K. Selective activation of neuromuscular compartments within the human trapezius muscle. J. Electromyogr. Kinesiol. 2009, 19, 896-902. [CrossRef] [PubMed]

25. Gosselin, G.; Rassoulian, H.; Brown, I. Effects of neck extensor muscles fatigue on balance. Clin. Biomech. 2004, 19, 473-479. [CrossRef] [PubMed]

26. Juul-Kristensen, B.; Kadefors, R.; Hansen, K.; Bystrom, P.; Sandsjo, L.; Sjogaard, G. Clinical signs and physical function in neck and upper extremities among elderly female computer users: The NEW study. Eur. J. Appl. Physiol. 2006, 96, 136-145. [CrossRef]

27. Falla, D.; Dall'Alba, P.; Rainoldi, A.; Merletti, R.; Jull, G. Location of innervation zones of sternocleidomastoid and scalene muscles-a basis for clinical and research electromyography applications. Clin. Neurophysiol. 2002, 113, 57-63. [CrossRef]

28. Jakobsen, M.D.; Sundstrup, E.; Andersen, C.H.; Zebis, M.K.; Mortensen, P.; Andersen, L.L. Evaluation of muscle activity during a standardized shoulder resistance training bout in novice individuals. J. Strength Cond. Res. 2012, 26, 2515-2522. [CrossRef]

29. Andersen, L.L.; Andersen, C.H.; Mortensen, O.S.; Poulsen, O.M.; Bjornlund, I.B.; Zebis, M.K. Muscle activation and perceived loading during rehabilitation exercises: Comparison of dumbbells and elastic resistance. Phys. Ther. 2010, 90, 538-549. [CrossRef]

30. Rivard, J.; Unsleber, C.; Schomacher, J.; Erlenwein, J.; Petzke, F.; Falla, D. Activation of the semispinalis cervicis and splenius capitis with cervical pulley exercises. Musculoskelet. Sci. Pract. 2017, 30, 56-63. [CrossRef]

31. Schomacher, J.; Erlenwein, J.; Dieterich, A.; Petzke, F.; Falla, D. Can neck exercises enhance the activation of the semispinalis cervicis relative to the splenius capitis at specific spinal levels? Man. Ther. 2015, 20, 694-702. [CrossRef] 
32. Gabriel, D.A.; Matsumoto, J.Y.; Davis, D.H.; Currier, B.L.; An, K.N. Multidirectional neck strength and electromyographic activity for normal controls. Clin. Biomech. 2004, 19, 653-658. [CrossRef]

33. Folland, J.P.; Williams, A.G. The adaptations to strength training: Morphological and neurological contributions to increased strength. Sports Med. 2007, 37, 145-168. [CrossRef]

34. Higbie, E.J.; Cureton, K.J.; Warren, G.L., 3rd; Prior, B.M. Effects of concentric and eccentric training on muscle strength, cross-sectional area, and neural activation. J. Appl. Physiol. 1996, 81, 2173-2181. [CrossRef]

35. Vikne, H.; Refsnes, P.E.; Ekmark, M.; Medbo, J.I.; Gundersen, V.; Gundersen, K. Muscular performance after concentric and eccentric exercise in trained men. Med. Sci. Sports Exerc. 2006, 38, 1770-1781. [CrossRef]

36. Jull, G.; Falla, D. Does increased superficial neck flexor activity in the craniocervical flexion test reflect reduced deep flexor activity in people with neck pain? Man. Ther. 2016, 25, 43-47. [CrossRef]

37. Aura, O.; Komi, P.V. Mechanical efficiency of pure positive and pure negative work with special reference to the work intensity. Int. J. Sports Med. 1986, 7, 44-49. [CrossRef]

38. Komi, P.V.; Kaneko, M.; Aura, O. EMG activity of the leg extensor muscles with special reference to mechanical efficiency in concentric and eccentric exercise. Int. J. Sports Med. 1987, 8 (Suppl. 1), 22-29. [CrossRef]

(C) 2020 by the authors. Licensee MDPI, Basel, Switzerland. This article is an open access article distributed under the terms and conditions of the Creative Commons Attribution (CC BY) license (http://creativecommons.org/licenses/by/4.0/). 